\title{
Asociación del polimorfismo del codon 72 del gen $p 53$ con el riesgo de cáncer gástrico en una población de alto riesgo de Costa Rica
}

\author{
Warner Alpízar-Alpízar ${ }^{1 *}$, Rafaela Sierra ${ }^{1}$, Patricia Cuenca $^{1}$, Clas Une $^{1}$, Fernando Mena $^{2}$ \& \\ Guillermo Ignacio Pérez-Pérez ${ }^{3}$ \\ 1 Instituto de Investigaciones en Salud (INISA), Universidad de Costa Rica, San José, Costa Rica. \\ 2 Sección de Patología, Hospital Dr. Max Peralta, Cartago, Costa Rica. \\ 3 Division of Infectious Diseases, New York University School of Medicine. New York, New York, United States of \\ America. \\ Correos electrónicos: (R. Sierra) rsierra@cariari.ucr.ac.cr; (P. Cuenca) pcuenca@cariari.ucr.ac.cr; \\ (C. Une) cune@ cariari.ucr.ac.cr; (F. Mena) fmenau@hmp.sa.cr; (G. Pérez-Pérez) perezg02@endeavor.med.nyu.edu \\ *Correspondencia: awarnercr@yahoo.com; warnera@ cariari.ucr.ac.cr; Tel.: (506)207-3294; Fax.: (506)207-5130 \\ Recibido 18-III-2005. Corregido 04-VII-2005. Aceptado 20-VII-2005.
}

\begin{abstract}
Association of the $p 53$ codon 72 polymorphism to gastric cancer risk in a hight risk population of Costa Rica. Gastric cancer is the second most common cancer associated death cause worldwide. Several factors have been associated with higher risk to develop gastric cancer, among them genetic predisposition. The p53 gene has a polymorphism located at codon 72 , which has been associated with higher risk of several types of cancer, including gastric cancer. The aim of this study was to determine the association of $p 53$, codon 72 polymorphism, with the risk of gastric cancer and pre-malignant lesions in a high-risk population from Costa Rica. The genotyping was carried out by PCR-RFLP in 58 gastric cancer patients, 99 controls and 41 individuals classified as group I or II, according to the Japanese histological classification. No association was found for p53, codon 72 polymorphism with neither the risk of gastric cancer nor the risk of less severe gastric lesions in the studied population. Based on this study and taking into account other studies carried out with p53, codon 72 polymorphism, the role of this polymorphism in the development of gastric cancer remains unclear. De novo mutations on $p 53$ gene produced during neoplasic development of this disease might play a greater role than germinal polymorphisms of the gene. Other polymorphic genes have been associated with higher risk to develop gastric cancer. Rev. Biol. Trop. 53(3-4): 317-324. Epub 2005 Oct 3.
\end{abstract}

Key words: Gastric cancer, $p 53$ polymorphism, codon 72, Helicobacter pylori, tumor suppressor gene.

El cáncer gástrico es la segunda causa de muerte por cáncer en el mundo, solo superado por el cáncer de pulmón (Parkin et al. 2001). Varios factores están asociados con el riesgo de desarrollar cáncer gástrico, entre ellos el bajo consumo de frutas y verduras frescas, la infección por Helicobacter pylori y la predisposición genética (Correa 1992, Kuipers 1999, Yasui et al. 2001, Correa 2003).

Uno de los genes que ha sido ampliamente asociado con cáncer es el gen que codifica la proteína supresora de tumores p53. Algunos estudios señalan que en más del $50 \%$ de todos los cánceres humanos, la proteína p53 no es funcional como resultado de mutaciones en el gen (Levine 1997, Vogelstein et al. 2000). El gen $p 53$ está localizado en el brazo corto del cromosoma 17 y codifica una fosfoproteína de 393 aminoácidos (Levine 1992, Walker y Levine 1996). Esta proteína ejerce su función como supresor de tumores mediante dos mecanismos, regulando de forma negativa el ciclo celular en la fase de transición de G1 a $\mathrm{S}$, o regulando de manera positiva la transcripción de genes relacionados con la regulación del ciclo celular. Además, desempeña un papel determinante en el proceso de apoptosis (Levine 1997). 
El gen p53 presenta algunas variantes polimórficas de línea germinal. Uno de dichos polimorfismos está localizado en el exón número cuatro, codón 72 del gen (Shepherd et al. 2000). Este consiste en una transversión de $\mathrm{G}$ por $\mathrm{C}$, lo cual da como resultado el cambio de un aminoácido en la secuencia de la proteína (arginina por prolina). Dicho cambio tiene efectos bioquímicos y biológicos importantes en cuanto a la acción de la proteína (Thomas et al. 1999). Este polimorfismo ha sido asociado con algunos tipos de cáncer entre ellos cáncer gástrico (Wang et al. 1999, Fan et al. 2000, Shepherd et al. 2000, Hiyama et al. 2002). Recientemente, este polimorfismo también ha sido asociado con la sobrevivencia de pacientes con cáncer gástrico (Zhang et al. 2003, Zhang et al. 2004).

Costa Rica es uno de los países con una de las más altas tasas de incidencia y mortalidad por cáncer gástrico a nivel mundial (Parkin et al. 2001). Se desconocen las causas de este fenómeno. La infección por $H$. pylori ha sido considerada como uno de los principales factores de riesgo de cáncer gástrico en el mundo, sin embargo en Costa Rica, la prevalencia de la infección por $H$. pylori es alta desde etapas tempranas de la vida, tanto en regiones de alto como de bajo riesgo del país (Sierra et al. 1992, Sierra et al. 1998). La infección por H. pylori, por si sola, no puede explicar las altas tasas de cáncer gástrico en Costa Rica, por lo que otros factores pueden estar involucrados, entre ellos factores relacionados con la dieta, el tipo de cepa de $H$. pylori, la respuesta del hospedero ante la infección por dicha bacteria y la susceptibilidad genética. En el presente estudio se investigó la asociación del polimorfismo del codón 72 , exón 4 del gen p53 con el riesgo de cáncer gástrico y lesiones gástricas en una población de alto riesgo de Costa Rica.

\section{MATERIALES Y MÉTODOS}

\section{Población de estudio}

El presente estudio es parte de un estudio más amplio $(n=1300$ personas $)$ sobre
"Marcadores para identificar sujetos en alto riesgo de cáncer gástrico" que se llevó a cabo en el Centro de Detección Temprana de Cáncer Gástrico de Costa Rica, entre los años 1999 y 2000. A partir de este proyecto, se utilizó la información epidemiológica y clínica de todos los pacientes con cáncer gástrico y de un grupo de pacientes con lesiones gástricas leves. Como grupo control se tomaron personas $\sin$ sospechas de cáncer por rayos $\mathrm{X}$ (serie gastroduodenal de doble contraste). Se formaron y compararon los siguientes grupos. A) 58 pacientes diagnosticados con cáncer gástrico mediante diagnóstico histopatológico (28 de tipo intestinal, 18 de tipo difuso y en 12 sin especificar); B) 58 personas sin sospecha de cáncer gástrico según el examen de rayos $\mathrm{X}$ (serie gastroduodenal de doble contraste) pareados por edad ( \pm 5 años) y sexo con respecto a los casos de cáncer; C) 41 personas con diagnóstico histopatológico de grupos I y II según la clasificación histológica Japonesa (Japanese Research Society for Gastric Cancer 1995); D) 41 personas no sospechosas de cáncer gástrico según el examen de rayos $\mathrm{X}$ (serie gastroduodenal de doble contraste), pareados por edad ( \pm 5 años) y sexo con respecto al grupo de lesiones gástricas leves (grupo C). El estudio fue aprobado por el comité ÉticoCientífico de la Universidad de Costa Rica. Todas las personas participantes habían firmado previamente una fórmula de consentimiento informado. A todos los individuos se les había tomado una muestra de sangre en el proyecto original, la cual estuvo almacenada a $-70^{\circ} \mathrm{C}$ hasta el momento de este estudio. Para el presente estudio se llevaron a cabo extracciones de ADN a partir de $0.5 \mathrm{ml}$ de dichas muestras de sangre mediante el protocolo de extracción con proteinasa-k y fenol/cloroformo.

\section{Determinación de los polimorfismos de p53}

La determinación de los polimorfismos del gen p53 se llevó a cabo mediante la reacción en cadena de la polimerasa para polimorfismos de restricción de diversos tamaños (PCR-RFLP). Los iniciadores y las condiciones de PCR 
fueron similares a las previamente descritas (Fan et al. 2000). El tamaño del producto final de amplificación fue de 199 pb. Volúmenes de $10 \mu \mathrm{l}$ de cada producto de amplificación fueron tratados con la enzima de restricción BstUI $\left(60^{\circ} \mathrm{C}\right.$ por 12 horas), posteriormente sometidos a electroforesis en geles de agarosa al $2 \%$ y teñidos con bromuro de etidio. El alelo que presenta la secuencia CGC en el exón 4 codón 72, la cual codifica el aminoácido Arginina (Arg), era digerido produciendo dos bandas de 133 y $86 \mathrm{pb}$, mientras que el alelo cuya secuencia es CCC, que codifica el aminoácido prolina (Pro), no era digerido por lo que se observaba una única banda de $199 \mathrm{pb}$. Los genotipos fueron los siguientes: CGC/CGC (Arg/Arg), dos bandas de 133 y 86 pb; CGC/CCC (Arg/Pro), tres bandas de 199, 133 y 86 pb; CCC/CCC (Pro/Pro), una única banda de 199 pb (Fan et al. 2000).

\section{Prevalencia de $H$. pylori}

La presencia de anticuerpos séricos contra $H$. pylori había sido determinada para cada uno de los pacientes del estudio original y estos resultados se utilizaron en el presente estudio. Dicha determinación se llevó a cabo mediante una prueba de ELISA (Enzyme Linked Immunosorbent Assay) desarrollada en nuestro laboratorio basado en un método descrito previamente (Pérez-Pérez et al. 1988, C. Une, no publicado). El antígeno fue preparado a partir de cinco cepas de $H$. pylori aisladas de pacientes de Costa Rica. 1001 del antígeno a una concentración de $10 \mathrm{~g} / \mathrm{ml}$ fueron colocados en cada uno de los pozos de placas para ELISA e incubados temperatura ambiente por una noche y posteriormente se adicionó 2501 de gelatina $0.1 \%$ en PBS y se incubó por tres horas a $37^{\circ} \mathrm{C}$ para bloquear cualquier adherencia inespecífica. Entre cada paso, las placas fueron lavadas tres veces en PBS + thimerosal $0.01 \%$ y Tween $200.05 \%$. Las muestras de suero usadas como controles negativos y positivos fueron diluidas 1:800 (7500 para los controles positivos) en PBS conteniendo gelatina $0.1 \%$ y gama-globulina bovina $0.5 \%$ (Sigma, USA), se agregaron 1001 de la dilución a cada uno de los pozos y se incubaron a $37^{\circ} \mathrm{C}$ durante sesenta minutos. Después del lavado, se agregaron 1001 de IgG humano conjugado con fosfatasa alcalina (Biosource International, USA). Las placas fueron incubadas durante sesenta minutos a $37^{\circ} \mathrm{C}$ y lavadas cinco veces. Posteriormente, se agregaron 1001 de sustrato (Fast p-Nitrophenyl Phosphate Tablet Sets, Sigma, USA) a las placas y fueron incubadas a $37^{\circ} \mathrm{C}$ hasta que la absorbancia neta a $405 \mathrm{~nm}$ excediera OD 0.4 para los controles positivos. La absorbancia fue determinada en un espectrofotómetro Multiscan (Thermo Labsystems, Finland). El punto de corte fue determinado a partir de los controles positivos usando la formula calculada a partir de una serie de experimentos con controles positivos y negativos. Los valores predictivos positivos y negativos fueron de $89 \%$ y $82 \%$ respectivamente.

\section{Análisis estadístico}

Se utilizó la prueba de Chi-cuadrado para comparar las frecuencias genotípicas del polimorfismo de p53 entre los grupos estudiados y para la determinación del equilibrio de Hardy-Weinberg. Se utilizó una prueba $t$ para comparar la edad promedio entre los grupos. Se calcularon los valores de Odd Ratio y modelos de regresión logística condicional para determinar posibles asociaciones entre el polimorfismo estudiado y cáncer gástrico utilizando el paquete estadístico STATA versión 8.1 (Stata Corporation, Texas, USA). En todas las pruebas calculadas, las diferencias fueron consideradas estadísticamente significativas para valores de $\mathrm{p} \leq 0.05$.

\section{RESULTADOS}

El cuadro 1 muestra las características generales de la población estudiada. Un alto porcentaje de las muestras estudiadas (87.4\%) fueron seropositivas para la prueba de anticuerpos anti-H. pylori. No se encontró diferencia en cuanto a la seropositividad para $H$. pylori entre el grupo de casos de cáncer gástrico y su 
CUADRO 1

Características de la población de estudio

\begin{tabular}{lcccc}
\multicolumn{1}{c}{ Diagnóstico } & $\begin{array}{c}\text { Edad promedio } \\
\pm \mathrm{DE}(\mathrm{años})\end{array}$ & $\begin{array}{c}\text { Hombres } \\
\mathrm{n}(\%)\end{array}$ & $\begin{array}{c}\text { Mujeres } \\
\mathrm{n}(\%)\end{array}$ & H. pylori $+(\%)$ \\
Con cáncer $\mathrm{n}=58$ & $59.8 \pm 13.8$ & $46(79.3)$ & $12(20.7)$ & $48(82.8)$ \\
Controles $\mathrm{n}=58$ & $61.4 \pm 9.2$ & $46(79.3)$ & $12(20.7)$ & $53(91.4)$ \\
Lesiones leves $\mathrm{n}=41$ & $64.3 \pm 8$ & $33(80.5)$ & $8(19.5)$ & $37(90.2)$ \\
Controles $\mathrm{n}=41$ & $65.7 \pm 5.6$ & $33(80.5)$ & $8(19.5)$ & $35(85.4)$ \\
TOTAL & 62.3 & $158(79.8)$ & $40(20.2)$ & $173(87.4)$
\end{tabular}

grupo control $\left(\chi^{2}=2.12, \mathrm{p}=0.15,2 \mathrm{gl}\right)$ ni entre el grupo de lesiones gástricas leves y su grupo control $\left(\chi^{2}=1.23, \mathrm{p}=0.27,2 \mathrm{gl}\right)$. Tampoco hubo diferencias significativas en la edad promedio al comparar los grupos estudiados. Debido al alto porcentaje de individuos con diagnóstico serológico positivo para $H$. pylori, no se consideró esta variable en los análisis estadísticos llevados a cabo.

El polimorfismo de p53 se encontró en equilibrio de Hardy-Weinberg en ambos grupos control (grupo control de los casos de cáncer gástrico y grupo control de las lesiones gástricas leves) $\left(\chi^{2}=1.88, \mathrm{p}=0.39\right.$ y $\chi^{2}=$ $0.25, \mathrm{p}=0.88$ respectivamente). El cuadro 2 muestra las frecuencias genotípicas y alélicas para el polimorfismo de $p 53$. No se observaron diferencias significativas al comparar las frecuencias genotípicas de los pacientes con cáncer versus su grupo control $\left(\chi^{2}=3.02\right.$, $\mathrm{p}=0.22$ ), ni al comparar las frecuencias genotípicas del grupo de lesiones gástricas leves versus sus respectivos controles $\left(\chi^{2}=0.80\right.$, $\mathrm{p}=0.67)$. Tampoco se encontraron diferencias al comparar las frecuencias genotípicas entre el grupo de casos de cáncer gástrico y el grupo de lesiones gástricas leves $\left(\chi^{2}=2.00, \mathrm{p}=0.37\right)$.

No se encontró asociación entre el polimorfismo del codón 72 de $p 53$ con el riesgo de cáncer gástrico (cuadro 3). Tampoco se observó asociación del polimorfismo de $p 53$ con el riesgo de desarrollar algún tipo de lesión gástrica leve. No se encontró asociación entre dicho polimorfismo y el riesgo de cáncer gástrico al utilizar como grupo control al grupo de lesiones leves y compararlos contra el grupo de pacientes con cáncer. No se determinó asociación del polimorfismo de $p 53$ con el riesgo de desarrollar cáncer gástrico de tipo intestinal, ni cáncer gástrico de tipo difuso (datos no mostrados).

\section{DISCUSIÓN}

En este estudio, un alto porcentaje de las personas estudiadas eran seropositivas para H. pylori $(87 \%)$. Estudios previos encontraron una prevalencia de infección muy alta en

CUADRO 2

Frecuencias genotípicas y alélicas para el polimorfismo de p53

$\begin{array}{cccccc}\text { Genotipo } & \text { Total } & \text { Cáncer } & \text { Controles } & \text { Lesiones leves } & \text { Controles } \\ & \mathrm{n}=175 & \mathrm{n}=47 & \mathrm{n}=47 & \mathrm{n}=40 & \mathrm{n}=41 \\ \text { Arg/Arg } & 97(0.55) & 27(0.57) & 26(0.55) & 21(0.52) & 23(0.56) \\ \text { Arg/Pro } & 60(0.35) & 16(0.34) & 17(0.36) & 14(0.35) & 13(0.32) \\ \text { Pro/Pro } & 18(0.10) & 4(0.09) & 4(0.09) & 5(0.13) & 5(0.12) \\ \text { Frecuencia alélica Pro } & 0.27 & 0.30 & 0.28 & 0.26 & 0.27\end{array}$


CUADRO 3

Valores de Odd Ratios e intervalos de confianza al 95\% para el polimorfismo de p53 en cáncer gástrico y lesiones graves

\begin{tabular}{lcccccr}
\multicolumn{1}{c}{ Genotipo } & $\begin{array}{c}\text { Cáncer gástrico } \\
\text { OR }(95 \% \text { IC) }\end{array}$ & $p \quad \begin{array}{c}\text { Lesiones leves } \\
\text { OR (95\% IC) }\end{array}$ & p & $\begin{array}{c}\text { Cáncer gástrico } \\
\text { OR (95\% IC) }\end{array}$ & $p$ \\
Arg/Arg & 1 (referente) & & 1 (referente) & & 1 (referente) & \\
Arg/Pro & $0.91(0.38-2.17)$ & 0.83 & $1.18(0.45-3.10)$ & 0.74 & $1.27(0.48-3.39)$ & 0.62 \\
Pro/Pro & $0.96(0.22-4.31)$ & 0.96 & $1.10(0.27-4.38)$ & 0.90 & $0.44(0.07-2.64)$ & 0.36 \\
Portadores de Pro & $0.92(0.40-2.08)$ & 0.84 & $1.16(0.48-2.79)$ & 0.75 & $1.04(0.42-2.60)$ & 0.93
\end{tabular}

pacientes dispépticos en la misma área geográfica (Morera-Brenes 1994, Sierra et al. 1998). En Costa Rica un alto porcentaje de la población está infectada con dicha bacteria desde edades muy tempranas (Sierra et al. 1991). Varios estudios han demostrado que $H$. pylori es uno de los principales factores de riesgo de cáncer gástrico y algunas cepas son más virulentas y han sido asociadas con un riesgo aun mayor de desarrollar cáncer gástrico (Huang et al. 2003). En este estudio no fue posible determinar el tipo de cepa de la bacteria presente en cada uno de los individuos de la muestra.

Las frecuencias alélicas del polimorfismo del exón 4 del gen p53 determinadas en nuestro estudio son similares a las informadas en varios estudios realizados en poblaciones de origen europeo (Fan et al. 2000, Zhang et al. 2003, Zhang et al. 2004). Sin embargo, estas difieren un poco de las frecuencias informadas por estudios llevados a cabo en Taiwán y Japón, donde la frecuencia del alelo asociado con el aumento en el riesgo de desarrollar varios tipos de cáncer (alelo Pro), es mayor a la encontrada en nuestro estudio (Wuang et al. 1999, Hiyama et al. 2002). Estudios previos han informado la existencia de algún grado de diferencia en cuanto a las frecuencias alélicas entre distintos grupos étnicos respecto al polimorfismo del exón 4 del gen p53 (Shepherd et al. 2000).

No se encontró ninguna asociación entre el polimorfismo del codón 72, exón 4 del gen p53 y el riesgo a desarrollar cáncer gástrico o lesiones leves en la población estudiada. Muy pocos estudios se han llevado a cabo para investigar la relación del polimorfismo del codón 72, exón 4 de p53 con el cáncer gástrico, sin embargo algunos de ellos han informado una asociación de dicho polimorfismo con aumento en el riesgo de desarrollar cáncer gástrico, tanto de tipo difuso como de tipo intestinal (Hiyama et al. 2002, Shen et al. 2004, Xi et al. 2004). Asimismo, se ha sugerido que la presencia del alelo Arg en este polimorfismo podría tener relación con el aumento en la sobrevivencia de pacientes con cáncer gástrico (Zhang et al. 2003, Zhang et al. 2004). Sin embargo, otros estudios no han encontrado asociación de dicho polimorfismo con el riesgo desarrollar esta enfermedad (Sheperd et al. 2000, Wu et al. 2004, Zhang et al. 2004). Por lo tanto, no está claro el papel que podría estar jugando el polimorfismo del codón 72, exón 4 del gen p53 en el desarrollo de cáncer gástrico.

Se sabe que el cambio de arginina (Arg) por prolina (Pro) en el codón 72 de la proteína p53 tiene efecto sobre la inducción de apoptosis y la activación de la transcripción (Thomas et al. 1999), sin embargo existen también otros polimorfismos en este gen, los cuales podrían estar relacionados con el aumento en el riesgo de cáncer gástrico (Shepherd et al. 2000). Asimismo, en el tejido transformado del cáncer gástrico se han encontrado una serie de mutaciones de novo en el gen p53 (Hsieh et al. 1996, Tolbert et al.1999, Shepherd et al. 2000). Muchas de estas mutaciones ocurren en las diferentes etapas del proceso neoplásico, por lo que algunas podrían tener un mayor efecto en el desarrollo tumoral que los polimorfismos de línea germinal existentes.

El cáncer gástrico es una enfermedad de etiología compleja y multifactorial. Muchos 
son los factores que han sido involucrados con aumento en el riesgo de desarrollar la enfermedad, desconocemos el peso que puede tener cada uno de ellos sobre el desarrollo de la enfermedad y sus efectos sobre los diferentes grupos étnicos y regiones geográficas. Existen otros genes polimórficos que han sido asociados con el riesgo de desarrollar cáncer gástrico, como por ejemplo genes que codifican interleucinas proinflamatorias y antiinflamatorias involucradas en la respuesta del hospedero ante la infección por H. pylori, (El-Omar et al. 2000, El-Omar et al. 2003). Asimismo, polimorfismos de genes relacionados con el metabolismo de sustancias carcinogénicas podrían estar relacionados con el desarrollo del cáncer gástrico (González et al. 2004).

\section{AGRADECIMIENTOS}

Esta investigación fue realizada con ayuda económica de la Vicerrectoría de Investigación de la Universidad de Costa Rica ( $\mathrm{N}^{\mathrm{o}}$ proyecto: 742-A2-142), el Ministerio de Ciencia y Tecnología de Costa Rica, el Consejo Nacional para Investigaciones Científicas Tecnológicas (CONICIT) y la Organización Panamericana de la Salud, y el Centro de Cooperación Científica de la Embajada de Francia. Agradecemos al Servicio Alemán de Intercambio Académico (DAAD) y al Organismo Internacional de Energía Atómica (OIEA) por la donación de equipo de laboratorio.

\section{RESUMEN}

El cáncer gástrico es la segunda causa de muerte por cáncer en el mundo. Varios factores han sido asociados con el riesgo de llegar a desarrollarlo, entre ellos la predisposición genética. El gen p53 presenta un polimorfismo en el codón 72, el cual ha sido asociado con un mayor riesgo de desarrollar varios tipos de cáncer entre ellos el gástrico. El objetivo de este estudio fue determinar la asociación del polimorfismo localizado en el codón 72 del gen p53 con el riesgo de cáncer gástrico y lesiones gástricas leves en una población de alto riesgo de Costa Rica. El análisis del polimorfismo se llevó a cabo mediante PCR-RFLP, en una muestra de 58 pacientes de cáncer gástrico, 99 personas controles y 41 individuos clasificados como grupos I y II de acuerdo con la clasificación histológica japonesa. No se determinó asociación del polimorfismo del codón 72 de $p 53$ con el riesgo de cáncer gástrico, ni de lesiones gástricas leves en la muestra estudiada. Con base en este estudio y otros que han investigado el polimorfismo del codón 72 del gen $p 53$, no está claro el papel que podría estar jugando dicho polimorfismo en el desarrollo de cáncer gástrico. Mutaciones de novo en el gen p53 producidas durante el desarrollo neoplásico de la enfermedad podrían tener un mayor efecto que polimorfismos de línea germinal de este mismo gen. Existen otros genes polimórficos que también se han asociado con el riesgo de desarrollar cáncer gástrico.

\section{REFERENCIAS}

Correa, P. 1992. Human gastric carcinogenesis: a multistep and multifactorial process First American Cancer Society Award Lecture on cancer epidemiology and prevention. Cancer Res. 52: 6735-6740.

Correa, P. 2003. Bacterial infections as a cause of cancer. J. Natl. Cancer Inst. 95: E3.

El-Omar, E., M. Carrington, W. Chow, K. McColl, J. Bream, H. Young, J. Herrera, J. Lissouska, C. Yuan, N. Rothman, G. Lanyon, M. Martin, J. Fraumeni \& C. Rabkin. 2000. Interleukin-1 polymorphisms associated with increased risk of gastric cancer. Nature 404: 398-406 (corrección publicada en Nature 2001; 412: 99).

El-Omar, E., C. Rabkin, M. Gammon, T. Vaughan, H. Risch, J. Schroenberg, J. Stanford, S. Mayne, J. Goedert, W. Blot, J. Fraumeni Jr \& W. Chow. 2003. Increased risk of noncardia gastric cancer associated with pro-inflammatory cytokine gene polymorphisms. Gastroenterology 124: 1193-1201.

Fan, R., M. Wu, D. Miller, J. Wain, K. Kelsey, J. Weincke \& D. Chistiani. 2000. The p53 codon 72 polymorphism and lung cancer risk. Cancer Epidemiol. Biomarkers Prev. 9: 1037-1042.

González, A., V. Ramírez, P. Cuenca \& R. Sierra. 2004. Polimorfismos en los genes de desintoxificación CYP1A1, CYP2E1, GSTT1 y GSTM1 en la susceptibilidad al cáncer gástrico. Rev. Biol. Trop. 52: 591-600.

Hiyama, T., S. Tanaka, Y. Kitadai, M. Ito, M. Sumii, M. Yoshihara, F. Shimamoto, K. Haruma \& K. Chayama. 2002. p53 codon polymorphism in gastric 
cancer susceptibility in patients with Helicobacter pylori-associated chronic gastritis. Int. J. Cancer 100: 304-308.

Hsieh, L.L., J.T. Hsieh, L.Y. Wang, C.Y. Fang, S.H. Chang \& T.C. Chen. 1996. p53 mutations in gastric cancer from Taiwan. Cancer Lett. 100: 107-113.

Huang, J.Q., G.F. Zheng, K. Sumanac, E.J. Irvine \& R. Hunt. 2003. Meta-analysis of the relationship between cagA seropositivity and gastric cancer. Gastroenterology 125: 1636-1644.

Japanese Research Society for Gastric Cancer. 1995. Japanese classification of gastric carcinoma. Tokio, Japan. 103 p.

Kuipers, E.J. 1999. Review article: exploring the link between Helicobacter pylori and gastric cancer. Aliment. Pharmacol. Ther. 13: 3-11.

Levine, A. 1992. The p53 tumor-suppressor gene. N. Engl. J. Med. 326: 1350-1352.

Levine, A. 1997. p53, the cellular gatekeeper for growth and division. Cell 88: 323-331.

Morera-Brenes, B., R. Sierra, R. Barrantes, J. Jonasson \& C. Nord. 1994. Helicobacter pylori in a Costa Rican dyspeptic patien population. Eur. J. Clin. Microbiol. Infect. Dis. 13: 253-257.

Parkin, M., F. Bray \& S. Devesa. 2001. Cancer burden in the year 2000. The global picture. Europ. J. Cancer 37: S4-S66.

Pérez-Pérez, G.I., B.M. Dworkin, J.E. Chodos \& M.J. Blaser. 1998. Campylobacter pylori antibodies in humans. Ann. Intern. Med. 109: 11-17.

Shepherd, T., D. Tolbert, J. Benedetti, J. MacDonald, G. Stemmermann, J. Wiest, G. DeVoe, M. Miller, J. Wang, A. Noffsinger \& C. Fenoglio-Preiser. 2000. Alterations in exon 4 of the p53 gene in gastric carcinoma. Gastroenterology 118: 1039-1044.

Shen, H., A. Solari, X. Wang, Z. Zhang, Y. Xu, L. Wang, X. Hu, J. Guo \& Q. Wei. 2004. P53 codon 72 polymorphism and risk of gastric cancer in a Chinese population. Oncol. Rep. 11: 1115-1120.

Sierra, R., D. Maxwell \& G. Muñoz. 1989. Cancer in Costa Rica. Cancer Res. 49: 717-724.

Sierra, R., N. Muñoz, A.S. Peña, I. Biemond, W. van Duijn, C.B.H.U. Lamers, S. Teuchmann, S. Hernandez \& P. Correa. 1992. Antibodies to Helicobacter pylori and pepsinogen levels in children from Costa Rica: comparison of two areas with different risks for stomach cancer. Cancer Epidemiol. Biomarkers Prev. 1: 449-454.

Sierra, R., P. Salas, F. Mora-Zuñiga, M. Sanabria, A. Chinnock, S. Peña, E. Quirós, W. Mora, F. Mena, R. Altman \& N. Muñoz. 1998. Erradicación de Helicobacter pylori en una población de alto riesgo de cáncer gástrico. Acta Med. Costarric. 40: 30-35.

Sierra, R., H. Ohshima, N. Muñoz, N. Teuchmann, A.S. Peña, C. Malaveille, B. Pignatelli, A. Chinnock, F. El Ghissassi, C. Chen, A. Hautefeuille, C. Gamboa \& H. Bartsch. 1991. Exposure to N-nitrosamines and other risk factors for gastric cancer in Costa Rican children. In Relevance to human cancer of $\mathrm{N}$-nitroso compounds, tobacco smoke and mycotoxins. L.K. O'Neill, J. Chen \& H. Bartsch (eds.). International Agency for Research on Cancer (IARC). Lyon, France.

Thomas, M., A. Kalita, S. Labrecque, D. Pim, L. Banks \& G. Matlasewski. 1999. Two polymorphic variants of wild type p53 differ biochemically and biologically. Mol. Cell. Biol. 19: 1092-1100.

Tolbert, D., C. Fenoglio-Preiser, A. Noffsinger, G. De Voe \& J. MacDonald. 1999. The relation of p53 gene mutations to gastric cancer subsite and phenotype. Cancer Causes Control 10: 227-231.

Vogelstein, B., D. Lane \& A. Levine. 2000. Surfing the p53 network. Nature 408: 307-310.

Walker, K. \& A. Levine. 1996. Identification of a novel p53 functional domain that is necessary for efficient growth suppression. Proc. Natl. Acad. Sci. 93: $15335-15340$.

Wang, Y.C., C.Y. Chen, S.K. Chen, Y.Y. Chang \& P. Lin. 1999. p53 codon 72 polymorphism in Taiwanese lung cancer patients: Association with lung cancer susceptibility and prognosis. Clin. Cancer Res. 5: 129-134.

Xi, Y.G., K.Y. Ding, X.L. Su, D.F. Chen, W.C. You, Y. Shen \& Y. Ke. 2004. p53 polymorphism and p $21^{\mathrm{WAF} 1 / \mathrm{CIP} 1}$ haplotype in the intestinal gastric cancer and precancerous lesions. Carcinogenesis 25: 2201-2206.

Yasui, W., N. Oue, H. Kuniyasu, R. Ito, R. Tahara \& H. Yokozaki. 2001. Molecular diagnosis of gastric cancer: present and future. Gastric Cancer 4: 113-121.

Zhang, Z.W., N. Laurence, A. Hollowood, P. Newcomb, M. Moorghen, J. Gupta, R. Feakins, M. Farthing, D. Alderson \& J. Holly. 2004. Prognostic value of TP53 codon 72 polymorphism in advanced gastric adenocarcinoma. Clin. Cancer Res. 10: 131-135. 
Zhang, Z.W., P. Newcomb, A. Hollowood, R. Feakins, M. Moorghen, A. Storey, M. Farthing, D. Alderson \& J. Holly. 2003. Age-associated increase of codon 72 arginine p53 frecuency in gastric cardia and non-cardia adenocarcinoma. Clin. Cancer Res. 9: 2151-2156.
Zhang, Z.W., P. Newcomb, A. Hollowood, Moganaden, J. Gupta, R. Feakins, A. Storey, M. Farthing, D. Alderson \& J. Holly. 2004. A comparison study of gastric cancer risk in patients with duodenal and gastric ulcer: roles of gastric mucosal histology and p53 codon 72 polymorphism. Dig. Dis. Sci. 49: 254-259. 